\title{
Malformation veineuse de la cavité buccale : à propos d'un
}

\section{cas.}

\author{
Saad $R^{1,2}$, Boatta $E^{3}$, Gaertner $S^{4}$, Riehm S \\ 1. Unité de Pathologie-Chirurgie buccale, Pôle de Médecine et de Chirurgie bucco-dentaires - HUS Strasbourg \\ 2. Faculté de Chirurgie Dentaire - Université de Strasbourg \\ 3. Unité de Radiologie Vasculaire et Interventionnelle, Pôle d'Imagerie - HUS Strasbourg \\ 4. Médecine Vasculaire - Les Hôpitaux Universitaires de Strasbourg (HUS) \\ 5. Service de Radiologie, Pôle d'Imagerie, Hôpital de Hautepierre - HUS Strasbourg \\ 6. Unité de Radiologie dento-maxillaire, Pôle de Médecine et de Chirurgie bucco-dentaires - HUS Strasbourg
}

\section{Introduction}

Les malformations artério-veineuses (MAV) sont des anomalies rares de la morphogénèse vasculaire, formées de vaisseaux artériels et veineux dysmorphiques interconnectés directement sans la transition d'un lit capillaire. Elles sont difficiles à prendre en charge, leur évolution est potentiellement péjorative et mettre en jeu le pronostic vital du patient par hémorragie massive (1).

\section{Observation}

Une patiente de 26 ans est adressée pour un avis concernant une lésion violacée papuleuse molle à la palpation siégeant dans le vestibule inférieur droit à l'aplomb de la dent $n^{\circ} 44$ en regard de la zone du foramen mentonnier et faisant environ $2 \mathrm{~cm}$ de diamètre. L'examen clinique en décubitus montrait une légère aggravation de la tuméfaction et un retour à la normale en station debout. L'OPT montrait en regard de cette lésion une image radioclaire bien circonscrite, superposée au foramen mentonnier et au contact de l'apex de 44. Une IRM injectée a confirmé l'existence d'une lésion qui se localisait à cheval dans les tissus mous de la muqueuse alvéolaire et de $12 \mathrm{~mm}$ de grand axe et, au sein de la médullaire mandibulaire avec également $12 \mathrm{~mm}$ de grand axe. Elle était hypointense en $\mathrm{T} 1$, hyperintense en $\mathrm{T} 2$ et se rehaussait progressivement après injection de gadolinium. Tous ces éléments étaient compatibles avec une malformation veineuse. Après avis auprès de la consultation spécialisée en radiologie interventionnelle et vasculaire, une abstention thérapeutique et un suivi annuel par IRM ont été décidés face à l'absence de signes fonctionnels, de la localisation et de la taille modérée de la lésion.

\section{Discussion}

La première anomalie décrite par le patient est en général une simple tache rosée ou une masse pulsatile. II peut se plaindre aussi d'une lourdeur sur le site, d'une douleur, d'un thrill, d'une hyperhidrose, d'une hypertrichose et enfin d'une hyperthermie (2). En 1998, une classification clinique des MAV proposée par R. Schobinger, établit 4 différents stades évolutifs de gravité croissante. L'examen radiologique standard ne donne aucun critère radiologique pathognomonique. L'échographie couplée au doppler couleur montre des tissus hyper-échogènes et une hypervascularisation tissulaire en cas d'extension extra-osseuse (1). Le scanner facial injecté et l'IRM précisent la localisation, l'extension et les rapports de la MAV. Mais c'est l'angiographie qui reste l'examen de choix, en analysant suffisamment l'architecture de la MAV et permettant ainsi d'orienter la meilleure approche thérapeutique.

La radiothérapie et les ligatures de la carotide externe et vasculaires ont laissé leur place à l'embolisation couplée ou non à un traitement chirurgical d'exérèse. La chirurgie ne sera réservée qu'aux formes évolutives ou compliquées (stades II à IV), mais il faudra y renoncer devant une lésion inextirpable qui est susceptible d'aggraver la MAV. Dans ces cas, l'embolisation peut être une alternative réduisant l'incidence des complications (3).

\section{Conclusion}

Ce cas est ainsi l'occasion de faire le point sur les MAV des maxillaires et montre que le chirurgien oral doit être avisé sur le dépistage de ces lésions et connaître leurs critères diagnostiques ainsi que les principes de leur prise en charge afin d'éviter tout accident hémorragique pouvant engager le pronostic vital.

fabien.bornert@chru-strasbourg.fr

(C) The authors, published by EDP Sciences. This is an Open Access article distributed under the terms of the Creative Commons Attribution License 4.0 (http://creativecommons.org/licenses/by/4.0/). 